\title{
Influence of Vitamin D Deficiency on Cardiometabolic Risk in Obesity
}

Edita Stokići ${ }^{*}$, Reza Hakkak ${ }^{2,3}$, Andrea Romani ${ }^{4}$, Aleksandar Kupusinac ${ }^{5}$, Esma Isenović ${ }^{6}$, Dragana Tomić-Naglić ${ }^{1}$, Biljana Srdić-Galicí ${ }^{7}$, Slađana Pejaković ${ }^{1}$ and Dragana Radoševic ${ }^{7}$

${ }^{1}$ Medical Faculty in Novi Sad, University of Novi Sad, Clinical Center of Vojvodina, Novi Sad, Serbia

${ }^{2}$ Department of Dietetics and Nutrition, University of Arkansas for Medical Sciences, Little Rock, AR 72205, USA

${ }^{3}$ Arkansas Children's Research Institute, Little Rock, AR 72202, USA

${ }^{4}$ Case Western Reserve university, Dept. Physiology and Biophysics, School of Medicine, 10900 Euclid Avenue, Cleveland, OH 44106-4970, USA

${ }^{5}$ Faculty of Technical Sciences, University of Novi Sad, Novi Sad, Serbia

${ }^{6}$ Laboratory for Molecular Genetics and Radiobiology, Institute of nuclear sciences "Vinca", University of Belgrade, Belgrade, Serbia

${ }^{7}$ Medical Faculty in Novi Sad, University of Vojvodina, Department of anatomy, Novi Sad, Serbia

Correspondence to:

Edita Stokić

Department of Endocrinology

Diabetes and Metabolic Diseases

Clinical Center of Vojvodina, 21000 Novi Sad

Hajduk Veljkova 1, Serbia

E-mail: edith@sezampro.rs

Received: January 20, 2017

Accepted: June 15, 2017

Published: June 18, 2017

Citation: Stokić E, Hakkak R, Romani A, Kupusinac A, Isenović E, et al. 2017. Influence of Vitamin D Deficiency on Cardiometabolic Risk in Obesity. J Obes Chronic Dis 1(2): 21-30.

Copyright: (C) 2017 Stokić et al. This is an Open Access article distributed under the terms of the Creative Commons Attribution 4.0 International License (CC-BY) (http://creativecommons.org/ licenses/by/4.0/) which permits commercial use, including reproduction, adaptation, and distribution of the article provided the original author and source are credited.

Published by United Scientific Group

\begin{abstract}
Vitamin D deficiency and dysfunctional adipose tissue are involved in the development of cardiometabolic disturbances (eg, hypertension, insulin resistance, type 2 diabetes mellitus, obesity, and dyslipidemia). We studied 50 obese (body mass index [BMI]: $43.5 \pm 9.2 \mathrm{~kg} / \mathrm{m}^{2}$ ) and 36 normal weight participants (BMI: $\left.22.6 \pm 1.9 \mathrm{~kg} / \mathrm{m}^{2}\right)$. Obese individuals were classified into different subgroups according to medians of observed anthropometric parameters (BMI, body fat percentage, waist circumference, and trunk fat mass). The prevalence of vitamin D deficiency (25-hydroxyvitamin D, $25(\mathrm{OH}) \mathrm{D}<50 \mathrm{nmol} / \mathrm{L})$ was $88 \%$ among obese patients and $31 \%$ among nonobese individuals; $25(\mathrm{OH}) \mathrm{D}$ were lower in the obese group $(27.3 \pm 13.7$ vs $64.6 \pm 21.3 \mathrm{nmol} / \mathrm{L}, \mathrm{p}<.001)$. There was a negative correlation between vitamin $\mathrm{D}$ and anthropometric indicators of obesity: BMI: $(\mathrm{r}=-0.64, \mathrm{p}<.001)$, waist circumference $(\mathrm{r}=-0.59 ; \mathrm{p}<.001)$, and body fat percentage $(r=-0.64 ; \mathrm{p}<.001)$ as well with fasting plasma insulin $(\mathrm{r}=-0.35 ; \mathrm{p}<$ $.001)$ and homeostasis model assessment of insulin resistance $(r=-0.35 ; p<.001)$. There was a negative correlation between vitamin $\mathrm{D}$ level and leptin and resistin $(\mathrm{r}=-.61 ; \mathrm{p}<.01)$, while a positive association with adiponectin concentrations were found $(\mathrm{r}=.7 ; \mathrm{p}<.001)$. Trend estimation showed that increase in vitamin $\mathrm{D}$ level is accompanied by intensive increase in adiponectin concentrations (growth coefficient: 12.13). In conclusion, we observed a higher prevalence of vitamin D deficiency among obese participants and this was associated with a proatherogenic cardiometabolic risk profile. In contrast, a positive trend was established between vitamin $\mathrm{D}$ and the protective adipocytokine adiponectin. The clinical relevance of this relationship needs to be investigated in larger studies.
\end{abstract}

\section{Keywords}

Obesity, Adipose tissue, Vitamin D

\section{Obesity}

According to the $11^{\text {th }}$ International Classification of Diseases, obesity is chronic non-infective disease [1]. It is characterized by an increase in fat mass to an extent that it may have an adverse effect on health, and occurs as a consequence of imbalance between energy intake and energy expenditure [2]. The prevalence of obesity is increasing worldwide as more than 1.9 billion adults are overweight and of these over 600 million were obese [3]. From the viewpoint of everyday clinical practice, calculation of body mass index (BMI) as the ratio of weight and height 
squared is a commonly used diagnostic tool in the detection of overweight and obesity in adults. Currently, obesity is defined as BMI greater than or equal to $30 \mathrm{~kg} / \mathrm{m}^{2}$ and $\mathrm{BMI}$ greater or equal to 25 is classified as overweight [4].

The diagnostic procedure in obese patients includes a number of parameters, with a focus on body fat distribution and metabolic profile of the person. Among anthropometric parameters significant are skinfolds, diameters and body circumferences. It has been shown that the measurement of sagittal abdominal diameter (SAD) is a good indicator of cardiovascular and metabolic risk [5]. Adipose mass distribution is very important for the assessment of obesity, because complications of obesity are caused mostly by visceral fat accumulation. The size of abdominal fat, especially its visceral depot, is responsible for the occurrence of insulin resistance, metabolic complications and cardiovascular diseases in obese [6]. Measurement of waist circumference is recommended to mark intra-abdominal fat depots and its correlates with cardiometabolic risk factors [6].

Obesity is characterized by a number of comorbidities such as type 2 diabetes mellitus, coronary heart disease, myocardial infarction, hypertension, atherogenic dyslipidemia, and osteoarthritis $[7,8]$. As a consequence of obesity, insulin resistance is an initial step towards to development of type 2 diabetes mellitus and most patients with diabetes are overweight [9]. In addition, it is estimated that $30 \%$ of obese people have lipid and lipoprotein disorders [10].

Adipose tissue produces several bioactive peptides, called adipocytokines, which are implicated in the complex pathogenesis of obesity-related metabolic disturbances.

\section{Dysfunctional Adipose Tissue}

Adipose tissue is considered as a major orchestrator of the obesity-related cardiometabolic pathophysiology. Recent investigations have revealed a number of different mechanisms by which adipose tissue takes part in the complex pathophysiology of insulin resistance, type 2 diabetes mellitus and cardiovascular disorders. Cardiometabolic consequences of obesity could be the result of the enlargement of adipose tissue mass (adiposity), and its adverse metabolic, endocrinologic and immunologic activities (adipose tissue dysfunction, adiposopathy, or "sick fat") [11-14].

Dysfunctional adipose tissue changes its standard endocrine pattern decreasing production of "defensive" adipokines such as adiponectin, and increasing secretion of "offensive" adipokines with pro-inflammatory, diabetogenic and proatherogenic properties [15-21]. The majority of pro-inflammatory adipokines is product of immune cells that populate adipose tissue [22]. Basically, obesity-linked complications can be considered as the result of the imbalance between pro- and anti-inflammatory adipokines. Hypoxia has been designated to be the key factor in the dysregulation of adipose tissue function; it induces secretion of key inflammation-related adipokines, including leptin and interleukin 6 (IL-6), and inhibits secretion of adiponectin which has anti-inflammatory and insulin-sensitising properties [13, 19, 23-25].
Overall, changes of adipose tissue microenvironment in obesity affect the metabolic and endocrine function of adipocytes enhancing development of low-grade inflammation and insulin resistance that are responsible for development of cardiometabolic complications.

\section{Vitamin D and Cardiometabolic Risk}

Serum concentrations of 25-hydroxyvitamin D, 25(OH) $\mathrm{D}$, are considered the best indicator of total body vitamin $\mathrm{D}$ stores, as value reflect total dietary intake and exposure to ultraviolet radiation [26]. Vitamin D deficiency is defined as 25(OH)D less than $50 \mathrm{nmol} / 1$ [26].

Today, there is evidence suggesting that vitamin D is a potential risk marker and modifiable risk factor for cardiovascular diseases [27, 28]. After 10-year followup, Giovannucci et al. found that men with vitamin D deficiency were at higher risk of myocardial infarction [28]. In addition, it was found that vitamin $\mathrm{D}$ deficiency is related to 2-fold increased cardiovascular risk among participants with hypertension [29]. Hence, the question is whether the determination of the degree of vitamin $\mathrm{D}$ deficiency can be used to estimate cardiometabolic risk? Also, several studies have proposed a relationship between vitamin $\mathrm{D}$ status and insulin resistance as they have found a negative association between vitamin $\mathrm{D}$ and fasting plasma insulin levels and homeostasis model assessment of insulin resistance (HOMA-IR) [30-33]. Because pancreatic $\beta$-cells express vitamin $D$ receptors, so a potential explanation is that vitamin $\mathrm{D}$ acts on these receptors [34] to stimulate pancreatic insulin secretion by regulating calcium entering into the $\beta$-cells [35]; thus, vitamin D may stimulate pancreatic insulin secretion. Additionally, in patients with type 2 diabetes mellitus, there is a suggestion that glucose intolerance improves after vitamin D supplementation [36, 37]. In relation to lipid profile, different results were obtained after vitamin $\mathrm{D}$ supplementation: Wamberg et al. failed to find any effects of increasing 25(OH)D levels on plasma lipids [38], while a study by Major et al. found that calcium and vitamin D supplementation decreased LDL-cholesterol levels [39]. As dyslipidemia is an important risk factor for cardiovascular diseases, there is a need for randomized controlled trials to clarify the possible effects of vitamin $\mathrm{D}$ on atherogenic dyslipidemia.

Adipose tissue stores energy, but the secretory products of adipocytes have been implicated in the pathogenesis of obesity-related metabolic disturbances. Namely, adipose tissue produces several bioactive peptides, known as adipocytokines, including leptin, TNF- $\alpha$, IL-6, adiponectin, and resistin. Proinflammatory and proatherogenic adipocytokines are involved in the development of insulin resistance, type 2 diabetes mellitus, and atherosclerosis [40]. It has been shown that in obese patients with dysfunctional adipose tissue, leptin levels are elevated, while a reduction in caloric intake is accompanied by reduced leptin concentrations [41]. Subcutaneous and visceral adipose tissue manifests different morphological and functional characteristics. In this regard, dysfunctional changes in adipose tissue are particularly expressed in visceral fat depots [42, 43], and individuals with 
predominantly excessive visceral adiposity are particularly vulnerable. It was found that $25(\mathrm{OH}) \mathrm{D}$ serum concentrations showed negative correlation with leptin levels [44, 45]. There is also evidence that $25(\mathrm{OH}) \mathrm{D}$ levels are positively associated with circulating adiponectin $[46,47]$.

Vitamin D deficiency has a trend to promote the development of a more proatherogenic cardiometabolic risk profile in obese patients. On the other hand, there is a need to investigate whether and to what extend vitamin D may improve adipose tissue function and thus prevent obesityrelated diseases. This is also the central theme of our research work. In this context, the goal of this study was to determine the vitamin $\mathrm{D}$ levels in obese and non-obese individuals and to assess the relationship between vitamin $\mathrm{D}$ and anthropometric measurements and cardiometabolic parameters.

\section{Materials and Methods}

\section{Patients and methods}

The inquired group consisted of 50 obese patients (BMI $\geq$ $30 \mathrm{~kg} / \mathrm{m}^{2}, 34$ women and 16 men with an average age of 38.2 \pm 11.3 years) and 36 normal weight participants (18 females and 18 males, average age $33.5 \pm 6.5$ and BMI from 18.5 to $25 \mathrm{~kg} / \mathrm{m}^{2}$. Table 1 presents characteristics of anthropometric parameters of study and control groups based on gender in form Mean \pm SD. The enquires were taken at the Department of Endocrinology, Diabetes and Metabolic Disorders, Clinical Centre of Vojvodina, Novi Sad, Serbia. Enquires were conducted according to the principles outlined in the Declaration of Helsinki. We excluded patients with recent weight changes and those who had been treated with vitamin $\mathrm{D}$ within 3 months prior, and also with previous history of beam-type balance to the nearest $0.1 \mathrm{~kg}$. In the standing position, body height $(\mathrm{BH})$ was measured using a Harpenden anthropometer (Holtain Ltd, Croswell, UK) with the precision of $0.1 \mathrm{~cm}$. The ration of body weight $(\mathrm{BW})$ and the square of body height $(\mathrm{BH})$ is body mass index (BMI). The flexible metric tape with the precision of $0.1 \mathrm{~cm}$ was used for measuring waist circumference (WC) at the level of middle distance between the lowest rib and the highest point on the iliac crest. The waist circumference correlates well with the size of the visceral abdominal adipose tissue, but also with the level of lipids, lipoproteins and insulin, and it is a significant predictor of the comorbidity of obesity [47-49]. BMI provides a measure that allows the comparison of the adiposity of individuals of different heights and weights, but does not provide sufficient information about fat mass and the relationship between FAT\% and BMI is gender- and age-dependent [50]. Body fat percentage (FAT\%) and trunk fat mass in $\mathrm{kg}$ (FAT trunk) was evaluated using bioelectrical impedance analysis (Tanita TBF310 Body Composition Analyzer: Tanita Corporation, Tokyo, Japan). During the anthropometric measurements, patients were without shoes and wearing light indoor clothing.

\section{Laboratory measurements}

The mercury sphygmomanometer was used for measuring systolic blood pressure (SBP) and diastolic blood pressure (DBP) in the sitting position after 10 to 15 minutes rest. Serum samples were obtained after an overnight fasting. The values of low-density lipoprotein cholesterol (LDL-C) were calculated using Friedewald equation [51]. The triglyceride (TG) levels were determined by an enzyme-based method and high-density lipoprotein cholesterol (HDL-C) was determined by the precipitation method with sodium phosphowolframate. Fasting plasma glucose, glucose oxidase

Table 1: Characteristics of anthropometric parameters of examined groups and in regard to gender.

\begin{tabular}{|c|c|c|c|c|c|c|}
\hline \multirow[b]{2}{*}{$\begin{array}{c}\text { Anthropometric } \\
\text { parameters }\end{array}$} & \multirow[b]{2}{*}{$\begin{array}{l}\text { Obese group } \\
\quad(n=50) \\
\text { Mean } \pm \text { SD }\end{array}$} & \multirow{2}{*}{$\begin{array}{l}\text { Control group } \\
\qquad(\mathrm{n}=36) \\
\text { Mean } \pm \text { SD } \\
\text { Obese }\end{array}$} & \multicolumn{2}{|c|}{ Women $(\mathrm{n}=52)$} & \multicolumn{2}{|c|}{$\operatorname{Men}(n=34)$} \\
\hline & & & $\begin{array}{c}\text { Obese } \\
(\mathrm{n}=34) \\
\text { Mean } \pm \text { SD }\end{array}$ & $\begin{array}{c}\text { Control } \\
(\mathrm{n}=18) \\
\text { Mean } \pm \mathrm{SD}\end{array}$ & $\begin{array}{c}\text { Obese } \\
(\mathrm{n}=16) \\
\text { Mean } \pm \mathrm{SD}\end{array}$ & $\begin{array}{c}\text { Control } \\
(\mathrm{n}=18) \\
\text { Mean } \pm \text { SD }\end{array}$ \\
\hline Age, years & $38.2 \pm 11.3$ & $33.5 \pm 6.5$ & $37.5 \pm 11.1$ & $34.3 \pm 7.9$ & $39.6 \pm 12.0$ & $32.8 \pm 4.7$ \\
\hline $\mathrm{SBP}, \mathrm{mmHg}$ & $133.0 \pm 20.5$ & $114.0 \pm 9.1$ & $131.0 \pm 19.8$ & $114.4 \pm 9.8$ & $137.2 \pm 21.9$ & $113.9 \pm 8.5$ \\
\hline $\mathrm{DBP}, \mathrm{mmHg}$ & $85.00 \pm 13.2$ & $78.0 \pm 4.6$ & $82.5 \pm 13.2$ & $78.1 \pm 5.5$ & $90.3 \pm 12.2$ & $78.3 \pm 3.8$ \\
\hline BW, kg & $126.8 \pm 29.1$ & $70.9 \pm 12.5$ & $117.4 \pm 26.0$ & $61.3 \pm 7.0$ & $146.7 \pm 25.6$ & $81.0 \pm 7.6$ \\
\hline $\mathrm{BMI}, \mathrm{kg} / \mathrm{m}^{2}$ & $43.5 \pm 9.2$ & $22.6 \pm 1.9$ & $42.4 \pm 9.4$ & $21.4 \pm 1.8$ & $45.9 \pm 8.7$ & $23.9 \pm 1.1$ \\
\hline FAT\% & $44.0 \pm 7.6$ & $21.8 \pm 6.4$ & $46.8 \pm 5.4$ & $25.4 \pm 5.4$ & $38.2 \pm 8.3$ & $17.7 \pm 5.0$ \\
\hline FAT trunk, $\mathrm{kg}$ & $26.7 \pm 7.9$ & $8.1 \pm 3.1$ & $25.6 \pm 6.6$ & $7.8 \pm 2.8$ & $29.3 \pm 10.3$ & $8.3 \pm 3.3$ \\
\hline $\mathrm{WC}, \mathrm{cm}$ & $128.5 \pm 20.4$ & $83.6 \pm 9.3$ & $122.8 \pm 18.9$ & $77.6 \pm 8.0$ & $140.7 \pm 18.4$ & $89.8 \pm 5.5$ \\
\hline
\end{tabular}

Abbreviations: SBP, systolic blood pressure; DBP, diastolic blood pressure; BW, body weight; BMI, body mass index; FAT\%, fat adipose tissue percentage; FAT trunk, trunk fat mass; WC, waist circumference; MEAN, Average value; SD, standard deviation.

diabetes mellitus, dyslipidemia, hypertension, heart, kidney, hepatic, malignant and psychiatric disorders, calcium level disturbances, Cushing's syndrome and thyroid dysfunction.

\section{Anthropometric measurements}

Body weight (BW) was determined using calibrated
- phenol + aminophenazone method, and fasting plasma insulin (FPI) levels by enzyme-linked immunosorbent assay. Homeostasis model assessment (HOMA-IR) was used to evaluate insulin resistance using the following formula: fasting glucose $(\mathrm{mmol} / \mathrm{L}) \mathrm{x}$ fasting insulin $(\mu \mathrm{U} / \mathrm{ml}) / 22.5$ [52]. An oral glucose tolerance test was performed to obtain glucose intake 
(2-hour plasma glucose and 2-hour plasma insulin).

ELISA (Enzyme-linked immunosorbent assay) method was used (enzyme immunoassay kit, Mediagnost, $\mathrm{GmbH}$ ) for the quantitative determination of serum leptin, resistin and adiponectin levels in $\mathrm{ng} / \mathrm{mL}$. As the best indicator of total body vitamin $\mathrm{D}$ stores, the serum concentrations of $25(\mathrm{OH}) \mathrm{D}$ were assessed using an enzyme immunotest (Immunodiagnostic system, United Kingdom). The deficiency of vitamin D was defined as $25(\mathrm{OH}) \mathrm{D}<50 \mathrm{nmol} / \mathrm{L}[53,54]$.

\section{Statistical analysis}

Programming environment MATLAB. 7.11.0 (Statistical toolbox) were used for data analysis-descriptive statistics, Mann Whitney test, linear correlation, linear regression and trend estimation with growth coefficients (GC). Data were expressed as Mean $\pm \mathrm{SD}$ and the $p$ values are classified as: < $.001^{* * *},<0.01^{* *},<0.05^{*}$, and $>.05$.

Obese individuals were classified into different subgroups with respect to previously calculated medians of anthropometric parameters: BMI median $\left(38.4 \mathrm{~kg} / \mathrm{m}^{2}\right)$, FAT\% median (45.1\%), WC median $(126.5 \mathrm{~cm})$ and FAT trunk median $(25.7 \mathrm{~kg})$. According to the median of the anthropometric parameters, obese patients were classified into two subgroups based on whether their values were larger or smaller than the median value. Linear correlations, regression lines and increasing $(\uparrow)$ or decreasing $(\downarrow)$ trends between vitamin $\mathrm{D}$ and adipocytokines within different subgroups were considered. Regression lines were obtained by using the method of least squares.

\section{Results}

Table 2 shows the differences between the obese and the control groups with respect to metabolic parameters. Nonobese participants had significantly lower FPI level and HOMA-IR $(\mathrm{p}<.001)$. Among lipid and lipoprotein parameters, HDL-C levels were significantly lower in the obese patients $(1.06 \pm$

Table 2: Metabolic parameters of study groups.

\begin{tabular}{|l|c|c|c|}
\hline \multirow{2}{*}{} & \multicolumn{2}{|c|}{ Groups } & \multirow{2}{*}{} \\
\cline { 2 - 3 } & Obese $(\mathrm{n}=50)$ & Control $(\mathrm{n}=36)$ & \multirow{2}{*}{$\mathrm{p}$} \\
\cline { 2 - 3 } & Mean $\pm \mathrm{SD}$ & Mean $\pm \mathrm{SD}$ & \multicolumn{1}{|c}{} \\
\hline $\mathrm{FPG}, \mathrm{mmol} / 1$ & $4.9 \pm 1.1$ & $4.7 \pm 0.4$ & $\mathrm{p}>.05$ \\
\hline $2 \mathrm{~h} \mathrm{PG}, \mathrm{mmol} / 1$ & $5.7 \pm 2.0$ & $5.0 \pm 1.2$ & $\mathrm{p}<.05^{*}$ \\
\hline FPI, mU/mL & $17.7 \pm 10.8$ & $6.7 \pm 4.2$ & $\mathrm{p}<.001^{* * *}$ \\
\hline $2 \mathrm{~h} \mathrm{PI}, \mathrm{mU} / \mathrm{mL}$ & $48.9 \pm 44.3$ & $23.4 \pm 21.1$ & $\mathrm{p}<.001^{* * *}$ \\
\hline HOMA-IR & $4.0 \pm 2.7$ & $1.4 \pm 1.0$ & $\mathrm{p}<.001^{* * *}$ \\
\hline $2 \mathrm{~h} \mathrm{HOMA}-\mathrm{IR}$ & $14.1 \pm 16.9$ & $5.9 \pm 6.9$ & $\mathrm{p}<.001^{* * *}$ \\
\hline LDL-C, mmol/1 & $3.6 \pm 1.0$ & $3.2 \pm 0.8$ & $\mathrm{p}>.05$ \\
\hline HDL-C, mmol/1 & $1.1 \pm 0.2$ & $1.4 \pm 0.3$ & $\mathrm{p}<.001^{* * *}$ \\
\hline TG, mmol/1 & $1.7 \pm 1.5$ & $1.2 \pm 0.9$ & $\mathrm{p}<.01$ \\
\hline
\end{tabular}

Abbreviations: FPG, fasting plasma glucose; 2h PG, 2-hour plasma glucose; FPI, fasting plasma insulin; 2h PI, 2-hour plasma insulin; HOMA-IR, homeostasis model assessment of insulin resistance; LDL-C, LDL cholesterol; HDL-C, HDL cholesterol; TG, triglycerides; 25(OH) D, 25-hydroxyvitamin D.
$0.23 \mathrm{mmol} / \mathrm{l})$ than in the nonobese participants $(1.42 \pm 0.31$ $\mathrm{mmol} / \mathrm{L} ; \mathrm{p}<.001)$.

The prevalence of vitamin D deficiency $(25(\mathrm{OH}) \mathrm{D}<$ $50 \mathrm{nmol} / \mathrm{L})$ was $88 \%$ among obese patients and $31 \%$ among nonobese individuals $(\mathrm{p}<.001)$. The mean serum 25(OH)D level was significantly lower in the obese group than in the control group $(27.3 \pm 13.7$ vs $64.6 \pm 21.3 \mathrm{nmol} / \mathrm{L} ; \mathrm{p}<.001 ;$ Figure 1$)$.

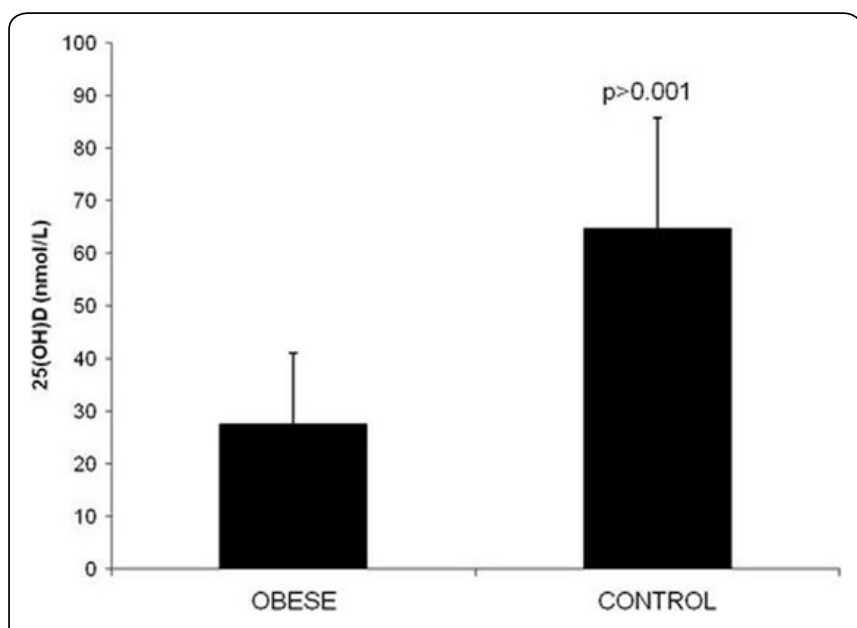

Figure 1: Mean serum 25(OH)D concentrations in the examined groups. $25(\mathrm{OH}) \mathrm{D}$ indicates 25 -hydroxyvitamin $\mathrm{D}$.

Table 3 shows that in all patients, coefficients of linear correlation between $25(\mathrm{OH}) \mathrm{D}$ levels and anthropometric parameters are negative and significant $(p<.001)$. A negative but slightly weaker correlation was also noted between serum $25(\mathrm{OH}) \mathrm{D}$ and SBP $(\mathrm{r}=-0.36 ; \mathrm{p}<.001)$ while there was no correlation with DBP $(r=-0.14 ; \mathrm{p}>.05)$.

Table 3: Coefficients of linear correlation $(r)$ between 25(OH)D level and examined anthropometric parameters for study patients

\begin{tabular}{|c|c|c|}
\hline & \multicolumn{2}{|c|}{ 25(OH)D } \\
\hline & $\mathbf{r}$ & $\mathbf{p}$ \\
\hline $\mathrm{BW}, \mathrm{kg}$ & -0.57 & $\mathrm{p}<.001^{* * * *}$ \\
\hline $\mathrm{BMI}, \mathrm{kg} / \mathrm{m}^{2}$ & -0.64 & $\mathrm{p}<.001^{* * *}$ \\
\hline $\mathrm{WC}, \mathrm{cm}$ & -0.59 & $\mathrm{p}<.001^{* * *}$ \\
\hline $\mathrm{SBP}, \mathrm{mmHg}$ & -0.36 & $\mathrm{p}<.001^{* * *}$ \\
\hline $\mathrm{DBP}, \mathrm{mmHg}$ & -0.14 & $p>.05$ \\
\hline FAT\% & -0.64 & $\mathrm{p}<.001^{* * * *}$ \\
\hline
\end{tabular}

Abbreviation: BW, body weight; BMI, body mass index; WC, waist circumference; SBP, systolic blood pressure; DBP, diastolic blood pressure; FAT\%, fat adipose tissue percentage; $25(\mathrm{OH}) \mathrm{D}, 25$-hydroxyvitamin D.

Results in Table 3A indicate that there is a negative coefficient of linear correlation between vitamin $\mathrm{D}$ and all observed anthropometric parameters, separately, both for women and for men.

Table 4 presents a negative correlation between vitamin $\mathrm{D}$ and FPI $(\mathrm{r}=-0.35 ; \mathrm{p}<.001)$ and HOMA-IR $(\mathrm{r}=-0.35 ; \mathrm{p}<$ $.001)$, while correlation with HDL-C was positive $(r=0.40$, $\mathrm{p}<.001)$. Vitamin D did not correlate with other metabolic parameters (glycaemia and lipids). 
Table 3A: Coefficients of linear correlation $(r)$ between 25(OH)D level and examined anthropometric parameters for study patients in relation to gender.

\begin{tabular}{|c|c|c|c|c|}
\hline \multirow{2}{*}{} & \multicolumn{4}{|c|}{$25(\mathrm{OH}) \mathrm{D}$} \\
\cline { 2 - 5 } & \multicolumn{2}{|c|}{ Women $(\mathrm{n}=52)$} & \multicolumn{2}{c|}{ Men $(\mathrm{n}=34)$} \\
\cline { 2 - 5 } & $\mathrm{r}$ & $\mathrm{p}$ & $\mathrm{r}$ & $\mathrm{p}$ \\
\hline $\mathrm{BW}, \mathrm{kg}$ & -0.63 & $<0.001^{* * *}$ & -0.71 & $<0.001^{\text {**** }}$ \\
\hline $\mathrm{BMI}, \mathrm{kg} / \mathrm{m}^{2}$ & -0.63 & $<0.001^{* * *}$ & -0.71 & $<0.001^{* * * *}$ \\
\hline $\mathrm{WC}, \mathrm{cm}$ & -0.63 & $<0.001^{* * *}$ & -0.69 & $<0.001^{\text {**** }}$ \\
\hline $\mathrm{SBP}, \mathrm{mmHg}$ & -0.37 & $<0.01^{\text {*** }}$ & -0.46 & $<0.01^{* *}$ \\
\hline $\mathrm{DBP}, \mathrm{mmHg}$ & -0.08 & $>0.05$ & -0.42 & $<0.05^{*}$ \\
\hline $\mathrm{FAT} \%$ & -0.66 & $<0.001^{* * *}$ & -0.61 & $<0.001^{\text {**** }}$ \\
\hline
\end{tabular}

Table 4: Coefficients of linear correlation $(r)$ between 25(OH)D level with metabolic parameters for all study patients.

\begin{tabular}{|c|c|c|}
\hline \multirow{2}{*}{ Correlation (N=86) } & \multicolumn{2}{|c|}{ 25(OH)D } \\
\cline { 2 - 3 } & $\mathbf{r}$ & $\mathbf{p}$ \\
\hline $\mathrm{FPG}, \mathrm{mmol} / \mathrm{l}$ & -0.19 & $\mathrm{p}>.05$ \\
\hline $2 \mathrm{~h} \mathrm{PG}, \mathrm{mmol} / 1$ & -0.17 & $\mathrm{p}>.05$ \\
\hline $\mathrm{FPI}, \mathrm{mIU} / \mathrm{L}$ & -0.35 & $\mathrm{p}<.001^{\text {**** }}$ \\
\hline $2 \mathrm{~h} \mathrm{PI}, \mathrm{mIU} / \mathrm{L}$ & -0.19 & $\mathrm{p}>.05$ \\
\hline $\mathrm{HOMA}-\mathrm{IR}$ & -0.35 & $\mathrm{p}<.001^{\text {**** }}$ \\
\hline $2 \mathrm{~h} \mathrm{HOMA}-\mathrm{IR}$ & -0.18 & $\mathrm{p}>.05$ \\
\hline $\mathrm{LDL}-\mathrm{C}, \mathrm{mmol} / 1$ & -0.10 & $\mathrm{p}>.05$ \\
\hline HDL-C, mmol/1 & 0.40 & $\mathrm{p}<.001^{\text {**** }}$ \\
\hline TG, mmol/1 & -0.09 & $\mathrm{p}>.05$ \\
\hline
\end{tabular}

Abbreviations: FPG, fasting plasma glucose; $2 \mathrm{~h}$ PG, 2-hour plasma glucose; FPI, fasting plasma insulin; 2h PI, 2-hour plasma insulin; HOMA-IR, homeostasis model assessment of insulin resistance; LDL-C, LDL cholesterol; HDL-C, HDL cholesterol; TG, triglycerides; 25(OH)D, 25-hydroxyvitamin D.

Table 5 lists linear equations that describe regression lines and trends: $\uparrow$ (increasing) and $\downarrow$ (decreasing). Linear equations were obtained by method of least squares. For almost all observed parameters, 25(OH)D level shows decreasing dependence, except for HDL-C.

The results in Table $5 \mathrm{~A}$ represents that vitamin $\mathrm{D}$ has a decreasing trend compared to all observed anthropometric parameters, based on gender.

Further, we assessed the relationship between vitamin D and adipose tissue dysfunction. According to the median of the anthropometric parameters (BMI, FAT\%, WC, and FAT trunk), obese patients were classified into 2 subgroups, those who have values larger and those who have values smaller than the median value.

Correlations between vitamin $\mathrm{D}$ and adipocytokines within different subgroups of BMI and FAT\% are presented in Table 6 . In the subgroup of patients with BMI $\geq 38.4 \mathrm{~kg} /$ $\mathrm{m}^{2}$, significant coefficients of linear correlation were obtained: negative with leptin and resistin $(\mathrm{r}=-.61, \mathrm{p}<0.01)$ and positive with adiponectin levels $(r=0.7, p<0.001)$. Vitamin
Table 5: Linear dependencies between all observed parameters and 25(OH)D.

\begin{tabular}{|c|c|c|}
\hline & \multicolumn{2}{|l|}{ 25(OH)D level } \\
\hline & Linear equations & Trends \\
\hline Body weight (BW), $\mathrm{kg}$ & $25(\mathrm{OH}) \mathrm{D}=-0.40 \times \mathrm{BW}+84.16$ & $\downarrow$ \\
\hline $\begin{array}{l}\text { Body mass index } \\
(\mathrm{BMI}), \mathrm{kg} / \mathrm{m}^{2}\end{array}$ & $25(\mathrm{OH}) \mathrm{D}=-1.29 \times \mathrm{BMI}+87.79$ & $\downarrow$ \\
\hline $\begin{array}{l}\text { Waist circumference } \\
\text { (WC), cm }\end{array}$ & $25(\mathrm{OH}) \mathrm{D}=-0.53 \times \mathrm{WC}+101.42$ & $\downarrow$ \\
\hline $\begin{array}{l}\text { Systolic blood pressure } \\
\text { (SBP), } \mathrm{mm} \mathrm{Hg}\end{array}$ & $25(\mathrm{OH}) \mathrm{D}=-0.47 \times \mathrm{SBP}+102.19$ & $\downarrow$ \\
\hline $\begin{array}{l}\text { Diastolic blood pressure } \\
\text { (DBP), } \mathrm{mm} \mathrm{Hg}\end{array}$ & $25(\mathrm{OH}) \mathrm{D}=-0.32 \times \mathrm{DBP}+69.23$ & $\downarrow$ \\
\hline $\begin{array}{c}\text { Body fat percentage, } \\
\text { BF\% }\end{array}$ & $25(\mathrm{OH}) \mathrm{D}=-1.20 \times \mathrm{BF} \%+84.45$ & $\downarrow$ \\
\hline $\begin{array}{l}\text { Fasting plasma glucose } \\
\text { (FPG), mmol/L }\end{array}$ & $25(\mathrm{OH}) \mathrm{D}=-5.56 \times \mathrm{FPG}+69.82$ & $\downarrow$ \\
\hline $\begin{array}{l}\text { 2-hour plasma glucose } \\
\text { (2h PG), mmol/L }\end{array}$ & $25(\mathrm{OH}) \mathrm{D}=-2.49 \times 2 \mathrm{~h} P \mathrm{PG}+56.42$ & $\downarrow$ \\
\hline $\begin{array}{l}\text { Fasting plasma insulin } \\
\text { (FPI), } \mathrm{mU} / \mathrm{mL}\end{array}$ & $25(\mathrm{OH}) \mathrm{D}=-0.86 \times \mathrm{FPI}+54.22$ & $\downarrow$ \\
\hline $\begin{array}{l}\text { 2-hour plasma insulin } \\
\text { (2h PI), } \mathrm{mU} / \mathrm{mL}\end{array}$ & $25(\mathrm{OH}) \mathrm{D}=-0.12 \times 2 \mathrm{~h} \mathrm{PI}+47.62$ & $\downarrow$ \\
\hline $\begin{array}{l}\text { Homeostasis model } \\
\text { assessment of insulin }\end{array}$ & $25(\mathrm{OH}) \mathrm{D}=-3.55 \times \mathrm{HOMA}-\mathrm{IR}+53.31$ & $\downarrow$ \\
\hline $\begin{array}{l}\text { 2-hour homeostasis } \\
\text { model assessment of }\end{array}$ & $25(\mathrm{OH}) \mathrm{D}=-0.32 \times 2 \mathrm{~h}$ HOMA-IR +46.31 & $\downarrow$ \\
\hline $\begin{array}{l}\text { LDL cholesterol } \\
\text { (LDL-C), mmol/L }\end{array}$ & $25(\mathrm{OH}) \mathrm{D}=-2.79 \times \mathrm{LDL}+52.38$ & $\downarrow$ \\
\hline $\begin{array}{l}\text { HDL cholesterol } \\
(\mathrm{HDL}-\mathrm{C}), \mathrm{mmol} / \mathrm{L}\end{array}$ & $25(\mathrm{OH}) \mathrm{D}=31.98 \times \mathrm{HDL}+4.20$ & $\uparrow$ \\
\hline $\begin{array}{c}\text { Triglycerides (TG), } \\
\mathrm{mmol} / \mathrm{L}\end{array}$ & $25(\mathrm{OH}) \mathrm{D}=-1.81 \times \mathrm{TG}+45.57$ & $\downarrow$ \\
\hline
\end{tabular}

Abbreviation: 25(OH)D, 25-hydroxyvitamin D.

Table 5A: Linear dependencies between all observed parameters and $25(\mathrm{OH}) \mathrm{D}$ respect to gender.

\begin{tabular}{|c|c|c|c|c|}
\hline & \multicolumn{4}{|c|}{ 25(OH)D level } \\
\hline & \multicolumn{2}{|c|}{ Women (n=52) } & \multicolumn{2}{|l|}{$\operatorname{Men}(n=34)$} \\
\hline & Linear equations & Trends & Linear equations & Trends \\
\hline $\mathrm{BW}, \mathrm{kg}$ & $\begin{array}{c}25(\mathrm{OH}) \mathrm{D}=- \\
0.45^{*} \mathrm{BW}+82.31\end{array}$ & $\downarrow$ & $\begin{array}{c}25(\mathrm{OH}) \mathrm{D}=- \\
0.47^{*} \mathrm{BW}+103.21\end{array}$ & $\downarrow$ \\
\hline BMI, $\mathrm{kg} / \mathrm{m}^{2}$ & $\begin{array}{c}25(\mathrm{OH}) \mathrm{D}=- \\
1.24^{*} \mathrm{BMI}+81.37\end{array}$ & $\downarrow$ & $\begin{array}{c}25(\mathrm{OH}) \mathrm{D}=- \\
1.40 * \mathrm{BMI}+98.63\end{array}$ & $\downarrow$ \\
\hline $\mathrm{WC}, \mathrm{cm}$ & $\begin{array}{c}25(\mathrm{OH}) \mathrm{D}= \\
-0.58 * \mathrm{WC}+100.28\end{array}$ & $\downarrow$ & $\begin{array}{c}25(\mathrm{OH}) \mathrm{D}=- \\
0.59^{*} \mathrm{WC}+118.65\end{array}$ & $\downarrow$ \\
\hline SBP, $\mathrm{mm} \mathrm{Hg}$ & $\begin{array}{c}25(\mathrm{OH}) \mathrm{D}=- \\
0.52^{*} \mathrm{SBP}+103.42\end{array}$ & $\downarrow$ & $\begin{array}{c}25(\mathrm{OH}) \mathrm{D}=- \\
0.58^{*} \mathrm{SBP}+123.26\end{array}$ & $\downarrow$ \\
\hline $\begin{array}{c}\mathrm{DBP}, \mathrm{mm} \\
\mathrm{Hg}\end{array}$ & $\begin{array}{c}25(\mathrm{OH}) \mathrm{D}=- \\
0.21 * \mathrm{DBP}+55.03\end{array}$ & $\downarrow$ & $\begin{array}{c}25(\mathrm{OH}) \mathrm{D}=- \\
1.02 * \mathrm{DBP}+136.82\end{array}$ & $\downarrow$ \\
\hline $\mathrm{BF} \%$ & $\begin{array}{c}25(\mathrm{OH}) \mathrm{D}=- \\
1.41^{*} \mathrm{FAT} \%+93.59\end{array}$ & $\downarrow$ & $\begin{array}{c}25(\mathrm{OH}) \mathrm{D}=- \\
1.23^{*} \mathrm{FAT} \%+84.64\end{array}$ & $\downarrow$ \\
\hline
\end{tabular}

D did not correlate with adipocytokines in other subgroups of anthropometric parameters (Table 7). 
Table 6: Coefficients of linear correlation $(r)$ between 25(OH)D Level and adipocytokines in subgroups of BMI and FAT\%.

\begin{tabular}{|c|c|c|c|c|}
\hline \multicolumn{5}{|c|}{ 25(OH)D, nmol/L } \\
\hline & \multicolumn{2}{|c|}{$\begin{array}{l}\text { BMI, Median, } \\
\mathrm{kg} / \mathrm{m}^{2}\end{array}$} & \multicolumn{2}{|c|}{ FAT $\%$, Median } \\
\hline & $<38.4$ & $\geq 38.4$ & $<45.1$ & $\geq 45.1$ \\
\hline \multirow{2}{*}{ Leptin, ng/mL } & $r=0.4$ & $r=-0.61$ & $r=-0.12$ & $r=-0.00$ \\
\hline & $p>.05$ & $\mathrm{p}<.01^{* *}$ & $p>.05$ & $p>.05$ \\
\hline \multirow{2}{*}{$\begin{array}{l}\text { Resistin, ng/ } \\
\mathrm{mL}\end{array}$} & $\mathrm{r}=0.5$ & $r=-0.6$ & $r=0.02$ & $r=-0.31$ \\
\hline & $p>.05$ & $\mathrm{p}<.05^{*}$ & $p>.05$ & $p>.05$ \\
\hline \multirow{2}{*}{$\begin{array}{l}\text { Adiponectin, } \\
\mathrm{ng} / \mathrm{mL}\end{array}$} & $r=0.1$ & $\mathrm{r}=0.7$ & $r=0.44$ & $r=-0.04$ \\
\hline & $\mathrm{p}>.05$ & $\mathrm{p}<.001^{* * * *}$ & $\mathrm{p}<0.5^{*}$ & $p>.05$ \\
\hline
\end{tabular}

Abbreviations: 25(OH)D, 25-hydroxyvitamin D; BMI, body mass index; FAT\%, fat adipose tissue percentage

Table 7: Coefficients of linear correlation $(r)$ between 25(OH)D Level and adipocytokines in subgroups of WC and FAT Trunk.

\begin{tabular}{|c|c|c|c|c|}
\hline \multicolumn{5}{|c|}{ 25(OH)D, $\mathbf{n m o l} / \mathbf{L}$} \\
\hline & \multicolumn{2}{|c|}{ WC, Median, cm } & \multicolumn{2}{c|}{ FAT trunk, Median, kg } \\
\hline & $<126.5$ & $\geq 126.5$ & $<25.7$ & $\geq 25.7$ \\
\hline \multirow{2}{*}{ Leptin, ng/mL } & $r=0.2$ & $r=-0.22$ & $r=-0.08$ & $r=-0.1$ \\
& $\mathrm{p}>.05$ & $\mathrm{p}>.05$ & $\mathrm{p}>.05$ & $\mathrm{p}>.05$ \\
\hline \multirow{2}{*}{ Resistin, ng/mL } & $\mathrm{r}=0.2$ & $\mathrm{r}=-0.35$ & $\mathrm{r}=0.03$ & $\mathrm{r}=-0.33$ \\
& $\mathrm{p}>.05$ & $\mathrm{p}>.05$ & $\mathrm{p}>.05$ & $\mathrm{p}>.05$ \\
\hline \multirow{2}{*}{ Adiponectin, ng/mL } & $\mathrm{r}=0.13$ & $\mathrm{r}=0.3$ & $\mathrm{r}=0.22$ & $\mathrm{r}=0.2$ \\
& $\mathrm{p}>.05$ & $\mathrm{p}>.05$ & $\mathrm{p}>.05$ & $\mathrm{p}>.05$ \\
\hline
\end{tabular}

Abbreviations: 25(OH)D, 25-hydroxyvitamin D; WC, waist

circumference; FAT trunk, trunk fat mass.

Tables 8 and 9 display linear equations that describe regression lines and increasing $(\uparrow)$ or decreasing $(\downarrow)$ trends between observed parameters: $25(\mathrm{OH}) \mathrm{D}$ and adipocytokines. The method of least squares was used to obtain linear equations. Within the subgroups of patients with higher degree of obesity (BMI $\geq 38.4 \mathrm{~kg} / \mathrm{m}^{2}$ and $\mathrm{FAT} \% \geq 45.1$ ), trend estimation shows inverse dependence between $25(\mathrm{OH})$ $\mathrm{D}$ level and leptin and resistin (Table 8). The same relationship was also noted in subgroups with WC and FAT trunk above the median - higher $25(\mathrm{OH}) \mathrm{D}$ is accompanied by decreasing trend of leptin and resistin (Table 9).

Observing all subgroups of obese patients, a positive trend was found between $25(\mathrm{OH}) \mathrm{D}$ level and adiponectin concentrations: with the higher $25(\mathrm{OH}) \mathrm{D}$ levels, adiponectin levels rise. The increase in adiponectin level was predominant among the subgroup of patients with $B M I \geq 38.4 \mathrm{~kg} / \mathrm{m}^{2}$, with GC of 12.13 (Table 8). Coefficient of adiponectin growth was also noticed in the subgroups of $\mathrm{WC} \geq 126.5 \mathrm{~cm}(\mathrm{GC}=4.3)$ and FAT trunk $\geq 25.7 \mathrm{~kg}(\mathrm{GC}=4)$ (Table 9).

\section{Discussion}

\section{Vitamin $\mathrm{D}$ and obesity}

Vitamin D deficiency is associated with cardiometabolic
Table 8: Linear dependencies and growth coefficients between adipocytokines and $25(\mathrm{OH}) \mathrm{D}$ within subgroups of $\mathrm{BMI}$ and FAT\% ${ }^{\mathrm{a}}$

\begin{tabular}{|c|c|c|c|}
\hline Subgroups & \multicolumn{3}{|c|}{ 25(OH)D, nmol/L } \\
\hline $\begin{array}{c}\mathrm{BMI}<38.4 \\
\mathrm{~kg} / \mathrm{m}^{2}\end{array}$ & Linear Equations & Trends & $\begin{array}{c}\text { Growth } \\
\text { Coefficient (GC) }\end{array}$ \\
\hline Leptin, $\mathrm{ng} / \mathrm{mL}$ & $0.55 \times \mathrm{LEP}+29.79$ & $\uparrow$ & 0.55 \\
\hline Resistin, ng/mL & $0.20 \times \mathrm{RES}+7.41$ & $\uparrow$ & 0.2 \\
\hline $\begin{array}{c}\text { Adiponectin, ng/ } \\
\mathrm{mL}\end{array}$ & $1.9 \times \mathrm{ADP}+1219$ & $\uparrow$ & 1.9 \\
\hline \multicolumn{4}{|l|}{$\mathrm{BMI} \geq 38.4 \mathrm{~kg} / \mathrm{m}^{2}$} \\
\hline Leptin, $\mathrm{ng} / \mathrm{mL}$ & $-0.79 \times$ LEP + 75.89 & $\downarrow$ & -0.79 \\
\hline Resistin, $\mathrm{ng} / \mathrm{mL}$ & $-0.21 \times \mathrm{RES}+22.28$ & $\downarrow$ & -0.21 \\
\hline $\begin{array}{l}\text { Adiponectin, } \mathrm{ng} / \\
\mathrm{mL}\end{array}$ & $12.13 \times \mathrm{ADP}+887.41$ & $\uparrow$ & 12.13 \\
\hline \multicolumn{4}{|l|}{ Fat $\%<45.1$} \\
\hline Leptin, ng/mL & $-0.16 \times$ LEP + 52.53 & $\downarrow$ & -0.16 \\
\hline Resistin, $\mathrm{ng} / \mathrm{mL}$ & $0.01 \times \mathrm{RES}+14.17$ & $\uparrow$ & 0.01 \\
\hline $\begin{array}{l}\text { Adiponectin, ng/ } \\
\mathrm{mL}\end{array}$ & $9.0 \times \mathrm{ADP}+1019.2$ & $\uparrow$ & 9 \\
\hline \multicolumn{4}{|l|}{ Fat $\% \geq 45.1$} \\
\hline Leptin, ng/mL & $-0.002 \times$ LEP +52.53 & $\downarrow$ & -0.002 \\
\hline Resistin, ng/mL & $-0.11 \times \mathrm{RES}+20.13$ & $\downarrow$ & -0.11 \\
\hline $\begin{array}{c}\text { Adiponectin, ng/ } \\
\mathrm{mL}\end{array}$ & $-0.6 \times \mathrm{ADP}+1186.1$ & $\downarrow$ & -0.6 \\
\hline
\end{tabular}

Abbreviations: 25(OH)D, 25-hydroxyvitamin D; BMI, body mass index; FAT\%, fat adipose tissue percentage; LEP, leptin; RES, resistin; ADP, adiponectin.

${ }^{\mathrm{a}}$ Growth coefficients describe the rate of increasing/decreasing of $25(\mathrm{OH})$ $\mathrm{D}$ level in relation to adipocytokines concentrations.

risk factors (e.g. hypertension, insulin resistance, type 2 diabetes mellitus, obesity, and dyslipidemia). In this context, there are reports of an association between obesity and vitamin D deficiency $[55,56]$. Many clinical and epidemiological studies reported that obese patients have lower serum concentrations of $25(\mathrm{OH}) \mathrm{D}$. In our study, the obese patients had significantly lower values of $25(\mathrm{OH}) \mathrm{D}$ than normal weight participants $(\mathrm{p}$ $<.001$ ). Consistent with prior studies, we found that vitamin $\mathrm{D}$ concentrations negatively correlated with BMI and WC [54-56]. Additionally, we also noted that fat mass is inversely associated with $25(\mathrm{OH}) \mathrm{D}$ levels which is in consistent with previous findings $[57,58]$.

After considering the linear dependence between anthropometric indicators of obesity (BMI, WC, and FAT\%) and 25(OH)D level, we found that for all mentioned parameters, the 25(OH)D level shows decreasing trends. With the increasing degree of obesity, vitamin D deficiency worsens. Since the BMI, as a practical measure of fatness, cannot distinguish fat mass from lean, we also took into consideration FAT\%. Using FAT content in body composition to determine obesity, our results show that a person with $30 \%$ body fat mass has a vitamin $\mathrm{D}$ level of $51.4136 \mathrm{nmol} / \mathrm{L}$ for females and $47.7475 \mathrm{nmol} / \mathrm{L}$ for males. Furthermore, our data indicate that vitamin $\mathrm{D}$ deficiency becames worst as body fat mass increases. 
levels $(\mathrm{r}=.7, \mathrm{p}<.001)$. This correlation became clearer after the evaluation of the trend, which showed that the increase in vitamin $\mathrm{D}$ level follows a similar upward trend adiponectin levels. The intensive increase in adiponectin was observed in the subgroup of BMI $>38.4 \mathrm{~kg} / \mathrm{m}^{2}$ with $\mathrm{GC}$ of 12.13 . This finding may be of importance in the context of therapeutic options for vitamin $\mathrm{D}$, since both the factors are implicated in the development of several cardiometabolic disturbances [47]. Also, numerous previous studies proved that intra-abdominal obesity has a direct impact on increased CV risk due to the increased action of proinflammatory and proatherogenic cytokines $[49,66]$. In our study, increase in adiponectin was also detected within the subgroups of patients who have larger WC and FAT trunk, with GCs of 4.3 and 4. This implies that changes in abdominal fat mass have an impact on the $25(\mathrm{OH})$ D-adiponectin relation.

\section{Conclusion}

In the present study, we report a higher prevalence of vitamin $\mathrm{D}$ deficiency among obese participants and a strong association between vitamin $\mathrm{D}$ and anthropometric measurements of obesity and fat distribution, fat mass, insulin resistance, HDL-C, and SBP. With higher vitamin $\mathrm{D}$ levels, almost all observed cardiometabolic parameters showed decreasing trend (except for HDL-C). Thus, vitamin $\mathrm{D}$ deficiency appears to promote the development of a more proatherogenic cardiometabolic risk profile in obese patients and individuals with increased fat mass. Measurement of vitamin $\mathrm{D}$ levels may help to identify individuals at greater cardiometabolic risk, and set the basis to design better intervention trials in obese patients.

Our results demonstrated negative correlation between $25(\mathrm{OH}) \mathrm{D}$ and leptin and resistin levels while a positive association with adiponectin concentrations was found. Linear dependence and trend between 25(OH)D level and leptin and resistin showed that with higher vitamin $\mathrm{D}$ levels, leptin and resistin have a downward trend. Further, trend estimation showed that increase in vitamin $\mathrm{D}$ level is accompanied by intensive increase in adiponectin concentrations.

In the view of present findings, we suggest that vitamin D supplementation may have a beneficial effect on obesity via modulation of adipocytokine secretions. Since the dysfunctional adipose tissue is a trigger for cardiometabolic disturbances in obese patients, interventional trials are required to establish whether vitamin D supplementation could be a therapeutic option for improving adipose tissue function and thus prevent obesity-related diseases.

\section{Acknowledgements}

This work was partially supported by the Ministry of Education, Science and Technological Development of the Republic of Serbia within the projects: ON 173033, ON 174026 and III 044006, and by the Provincial Secretariat for Science and Technological Development of the Autonomous Province of Vojvodina within the project: 114-451-2856/2016.

\section{References}

1. The International Classification of Diseases, $11^{\text {th }}$ revision. 2012. World Health Organization.

2. Stokić E. 2004. [Obesity is a disease that is treated. Novi Sad, Faculty of Medicine, University of Novi Sad].

3. WHO. 2011. Obesity and overweight, Fact sheet $\mathrm{N}^{\circ} 311$.

4. Political Declaration, UN high-level meeting of the general assembly on the prevention and control of non-communicable diseases, September 2011, A/66/L.1

5. Ohrvall M, Berglund L, Vessby B. 2000. Sagittal abdominal diameter compared with other anthropometric measurements in relation to cardiovascular risk. Int J Obes Relat Metab Disord 24(4): 497-501.

6. Marques-Vidal P, Pécoud A, Hayoz D, Paccaud F, Mooser V, et al. 2010. Normal weight obesity: relationship with lipids, glycaemic status, liver enzymes and inflammation. Nutr Metab Cardiovasc Dis 20(9): 669-675. doi: https://doi.org/10.1016/j.numecd.2009.06.001.

7. Must A, Spadano J, Coakley EH, Field AE, Colditz G, et al. 1999. The disease burden associated with overweight and obesity. JAMA 282(16): 1523-1529. doi: https://doi.org/10.1001/jama.282.16.1523

8. National Health and Medical Research Council. 2013. Summary guide for the management of overweight and obesity in primary care. Australia.

9. Marinou K, Tousoulis D, Antonopoulos A, Stefanadi E, Stefanadis C. 2010. Obesity and cardiovascular disease: from pathophysiology to risk stratification. Int J Cardiol 138(1): 3-8. doi: https://doi.org/10.1016/j. ijcard.2009.03.135

10. Stokic E,Tomic-Naglic D, Djeric M,Jorga J. 2009. [Therapeutic options for treatment of cardiometabolic risk]. Med Pregl 62 Suppl 3: 54-58.

11. Bays H, Abate N, Chandalia M. 2005. Adiposopathy: sick fat causes high blood sugar, high blood pressure, and dyslipidemia. Future Cardiol (1)1: 39-59. doi: https://doi.org/10.1517/14796678.1.1.39

12. Bays H, Ballantyne C. 2006. Adiposopathy: why do adiposity and obesity cause metabolic disease? Future Lipidol 1(4): 389-420.

13. Bays HE, Gonzalez-Campoy JM, Henry RR, Bergman DA, Kitabchi $\mathrm{AE}$, et al. 2008. Is adiposopathy (sick fat) an endocrine disease? Int J Clin Pract 62(10): 1474-1483. doi: https://doi.org/10.1111/j.17421241.2008.01848.x

14. Bays HE. 2011. Adiposopathy is "sick fat" a cardiovascular disease? $J$ Am Coll Cardiol 57(25): 2461-2473. doi: https://doi.org/10.1016/j. jacc.2011.02.038

15. Blüher M. 2012. Are there still healthy obese patients? Curr Opin Endocrinol Diabetes Obes 19(5): 341-346. doi: https://doi.org/10.1097/ MED.0b013e328357f0a3

16. Klöting N, Fasshauer M, Dietrich A, Kovacs P, Schön MR, et al. 2010. Insulin-sensitive obesity. Am J Physiol Endocrinol Metab 299(3): E506-E515. doi: https://doi.org/10.1152/ajpendo.00586.2009

17. Goossens GH. 2008. The role of adipose tissue dysfunction in the pathogenesis of obesity-related insulin resistance. Physiol Behav 94(2): 206-218. doi: https://doi.org/10.1016/j.physbeh.2007.10.010

18. Hardy OT, Perugini RA, Nicoloro SM, Gallagher-Dorval K, Puri V, et al. 2011. Body mass index-independent inflammation in omental adipose tissue associated with insulin resistance in morbid obesity. Surg Obes Relat Dis 7(1): 60-67. doi: https://doi.org/10.1016/j. soard.2010.05.013

19. Srdić B, Stokić E, Korać A, Ukropina M, Veličković K, et al. 2010. Morphological characteristics of abdominal adipose tissue in normal-weight and obese women of different metabolic profiles. Exp Clin Endocrinol Diabetes 118(10): 713-718. doi: https://doi. org/10.1055/s-0030-1254165

20. Slawik M, Vidal-Puig A. 2007. Adipose tissue expandability and metabolic syndrome. Genes Nutr 2: 41-45. doi: https://doi.org/10.1007/ s12263-007-0014-9 
21. Fain JN. 2006. Release of interleukins and other inflammatory cytokines by human adipose tissue is enhanced in obesity and primarily due to the nonfat cells. Vitam Horm 74: 443-477. doi: https://doi.org/10.1016/ S0083-6729(06)74018-3

22. Trayhurn P, Wang B, Wood IS. 2008. Hypoxia and the endocrine and signalling role of white adipose tissue. Arch Physiol Biochem 114: 267276. doi: https://doi.org/10.1080/13813450802306602

23. Kim KH, Song MJ, Chung J, Park H, Kim JB. 2005. Hypoxia inhibits adipocyte differentiation in a HDAC-independent manner. Biochem Biophys Res Commun 333(4): 1178-1184. doi: https://doi.org/10.1016/j. bbrc.2005.06.023

24. Kim JI, Huh JY, Sohn JH, Choe SS, Lee YS, et al. 2015. Lipidoverloaded enlarged adipocytes provoke insulin resistance independent of inflammation. Mol Cell Biol 35(10): 1686-1699. doi: https://doi. org/10.1128/MCB.01321-14

25. Holick MF. 2007. Vitamin D deficiency. N Engl J Med 357: 266-281. doi: https://doi.org/10.1056/NEJMra070553

26. Dobnig H, Pilz S, Scharnagl H, Renner W, Seelhorst U, et al. 2008. Independent association of low serum 25-hydroxyvitamin D and 1,25-dihydroxyvitamin D levels with all-cause and cardiovascular mortality. Arch Intern Med 168(12): 1340-1349. doi: https://doi. org/10.1001/archinte.168.12.1340

27. Reis JP, von Mühlen D, Miller ER $3^{\text {rd }}$, Michos ED, Appel LJ. 2009. Vitamin D status and cardiometabolic risk factors in the United States adolescent population. Pediatrics 124(3): e371-e379. doi: https://doi. org/10.1542/peds.2009-0213

28. Giovannucci E, Liu Y, Hollis BW, Rimm EB. 2008. 25-hydroxyvitamin $\mathrm{D}$ and risk of myocardial infarction in men: a prospective study. Arch Intern Med 168(11): 1174-1180. doi: https://doi.org/10.1001/ archinte.168.11.1174

29. Wang TJ, Pencina MJ, Booth SL, Jacques PF, Ingelsson E, et al. 2008. Vitamin D deficiency and risk of cardiovascular disease. Circulation 117(4): 503-511. doi: https://doi.org/10.1161/ CIRCULATIONAHA.107.706127.

30. Chiu KC, Chu A, Go VLW, Saad M. 2004. Hypovitaminosis D is associated with insulin resistance and $\beta$ cell dysfunction. Am J Clin Nutr 79(5): 820-825.

31. Heaney RP, French CB, Nguyen S, Ferreira M, Baggerly LL, et al. 2013. A novel approach localizes the association of vitamin $D$ status with insulin resistance to one region of the 25 -hydroxyvitamin $\mathrm{D}$ continuum. Adv Nutr 4(3): 303-310. doi: https://doi.org/10.3945/an.113.003731

32. Schöttker B, Herder C, Rothenbacher D, Perna L, Müller H, et al. 2013. Serum 25-hydroxyvitamin D levels and incident diabetes mellitus type 2: a competing risk analysis in a large population-based cohort of older adults. Eur J Epidemiol 28(3): 267-275. doi: https://doi.org/10.1007/ s10654-013-9769-z

33. Tai K, Need AG, Horowitz M, Chapman IM. 2008. Vitamin D, glucose, and insulin sensitivity. Nutrition 24(3): 279-285. doi: https:// doi.org/10.1016/j.nut.2007.11.006

34. Pittas AG, Lau J, Hu FB, Dawson-Hughes B. 2007. Review: the role of vitamin $\mathrm{D}$ and calcium in type 2 diabetes. A systematic review and meta-analysis. J Clin Endocrinol Metab 92(6): 2017-2029. doi: https:// doi.org/10.1210/jc.2007-0298

35. Hutchinson MS, Figenschau Y, Almås B, Njølstad I, Jorde R. 2011. Serum 25-hydroxyvitamin D levels in subjects with reduced glucose tolerance and type 2 diabetes - the Tromsø OGTT-study. Int J Vitam Nutr Res 81(5): 317-327. doi: https://doi.org/10.1024/0300-9831/ a000079

36. Nazarian S, St Peter JV, Boston RC, Jones SA, Mariash CN. 2011. Vitamin D3 supplementation improves insulin sensitivity in subjects with impaired fasting glucose. Transl Res 158(5): 276-281. doi: https:// doi.org/10.1016/j.trsl.2011.05.002

37. Wamberg L, Kampmann U, Stødkilde-Jørgensen H, Rejnmark L,
Pedersen SB, et al. 2013. Effects of vitamin D supplementation on body fat accumulation, inflammation, and metabolic risk factors in obese adults with low vitamin D levels - results from a randomized trial. Eur J Intern Med 24(7): 644-649. doi: https://doi.org/10.1016/j. ejim.2013.03.005

38. Major GC, Alarie F, Dore J, Phouttama S, Tremblay A. 2007. Supplementation with calcium vitamin $\mathrm{D}$ enhances the beneficial effect of weight loss on plasma lipid and lipoprotein concentrations. Am J Clin Nutr 85(1): 54-59.

39. Murdolo G, Smith U. 2006. The dysregulated adipose tissue: a connecting link between insulin resistance, type 2 diabetes mellitus and atherosclerosis. Nutr Metab Cardiovasc Dis 16 suppl 1: S35-S38. doi: https://dx.doi.org/10.1016/j.numecd.2005.10.016

40. Kershaw EE, Flier JS. 2004. Adipose tissue as an endocrine organ. $J$ Clin Endocrinol Metab 89(6): 2548-2556. doi: https://doi.org/10.1210/ jc.2004-0395

41. Misra A, Vikram NK. 2003. Clinical and pathophysiological consequences of abdominal adiposity and abdominal adipose tissue depots. Nutrition 19(5): 457-466. doi: https://dx.doi.org/10.1016/ S0899-9007(02)01003-1

42. Stokić E, Srdić-Galić B, Tomić-Naglić D, Isenović E. 2013. Sagittal abdominal diameter (SAD) in identification obese patients of higher cardiovascular risk. Cardiology 126: 169.

43. Vilarrasa N, Vendrell J, Maravall J, Elio I, Solano E, et al. 2010. Is plasma $25(\mathrm{OH}) \mathrm{D}$ related to adipokines, inflammatory cytokines and insulin resistance in both a healthy and morbidly obese population? Endocrine 38(2): 235-242. doi: https://doi.org/10.1007/s12020-010-9379-4

44. Kempt Am SM, Li C, Kaur H, Huang Tt. 2006. Leptin is a marker of body fat and hyperinsulinemia in college students. J Am Coll Nutr 55(3): 175-180. doi: https://dx.doi.org/10.3200/JACH.55.3.175-180

45. Kim M, Na W, Sohn C. 2013. Correlation between vitamin D and cardiovascular disease predictors in overweight and obese Koreans. J Clin Biochem Nutr 52(2): 167-171. doi: https://doi.org/10.3164/ jcbn.12-81

46. Liu E, Meigs JB, Pittas AG, McKeown NM, Economos CD, et al. 2009. Plasma 25-hydroxyvitamin D is associated with markers of the insulin resistant phenotype in non-diabetic adults. J Nutr 139(2): 329334. doi: https://doi.org/10.3945/jn.108.093831

47. Kupusinac A, Stokić E, Srdić-Galić B. 2012. Determination of WHtR limit for predicting hyperglycemia in obese persons by using artificial neural networks. TEMJ1(4): 271-272.

48. Vaidya A, Williams JS, Forman JP. 2012. The independent association between 25-hydroxyvitamin D and adiponectin and its relation with BMI in two large cohorts: the NHS and the HPFS. Obesity(Silver Spring) 20(1): 186-191. doi: https://doi.org/10.1038/oby.2011.210

49. Friedewald WT, Levy RI, Fredrickson DS. 1972. Estimation of the concentration of low-density lipoprotein cholesterol in plasma, without use of the preparative ultracentrifuge. Clin Chem 18(6): 499-502.

50. Maththews DR, Hosker JP, Rudenski AS, Naylor BA, Treacher DF, et al. 1985. Homeostasis model assessment: insulin resistance and betacell function from fasting plasma glucose and insulin concentrations in man. Diabetologia 28(7): 412-419.

51. Stokić E, Kupusinac A, Tomić-Naglić D, Kovačev-Zavišić B, Mitrović $\mathrm{M}$, et al. 2014. Obesity and vitamin D deficiency: trends to promote a more proatherogenic cardiometabolic risk profile. Angiology 66(3): 237243. doi: https://doi.org/10.1177/0003319714528569

52. Taheri E,Saedisomeolia A,Djalali M, Qorbani M,Madani Civi M.2012. The relationship between serum 25-hydroxy vitamin D concentration and obesity in type 2 diabetic patients and healthy subjects. J Diabetes Metab Disord 11(1): 16. doi: https://doi.org/10.1186/2251-6581-11-16.

53. Liel Y, Ulmer E, Shary J, Hollis BW, Bell NH. 1988. Low circulating vitamin D in obesity. Calcif Tissue Int 43(4): 199-201.

54. Hyldstrup L, Andersen T, Mcnair P, Breum L, Transbol I. 1993. Bone 
metabolism in obesity: changes related to severe overweight and dietary weight reduction. Acta Endocrinol (Copenh.) 129(5): 393-398. doi: https://doi.org/10.1530/acta.0.1290393

55. Bischoff-Ferrari HA, Giovannucci E, Willett WC, Dietrich T, Dawson-Hughes B. 2006. Estimation of optimal serum concentrations of 25-hydroxyvitamin D for multiple health outcomes. Am J Clin Nutr 84(1): $18-28$

56. De Pergola G, Nitti A, Bartolomeo N, Gesuita A, Giagulli VA, et al. 2013. Possible role of hyperinsulinemia and insulin resistance in lower vitamin D levels in overweight and obese patients. Biomed Res Int 921348. doi: https://doi.org/10.1155/2013/921348.

57. Buffington C, Walker B, Cowan GS Jr, Scruggs D. 1993. Vitamin D deficiency in the morbidly obese. Obes Surg 3(4): 421-424. doi: https:// doi.org/10.1381/096089293765559142

58. Botella-Carretero JI, Alvarez-Blasco F, Villafruela JJ, Balsa JA, Vazquez $\mathrm{C}$, et al. 2007. Vitamin D deficiency is associated with the metabolic syndrome in morbid obesity. Clin Nutr 26(5): 573-580. doi: http:// dx.doi.org/10.1016/j.clnu.2007.05.009

59. Pilz S, Tomaschitz A, Ritz E, Pieber TR. 2009. Vitamin D status and arterial hypertension: a systematic review. Nat Rev Cardiol 6(10): 621630. doi: https://doi.org/10.1038/nrcardio.2009.135

60. Al Masri M, Romain AJ, Boegner C, Maimoun L, Mariano-Goulart D, et al. 2017. Vitamin D status is not related to insulin resistance in different phenotypes of moderate obesity. Appl Physiol Nutr Metab 42(4): 438-442. doi: https://doi.org/10.1139/apnm-2016-0298

61. Cheng S, Massaro JM, Fox CS, Larson MG, Keyes MJ, et al. 2010 Adiposity, cardiometabolic risk, and vitamin D status: the Framingham Heart Study. Diabetes 59(1): 242-248. doi: https://doi.org/10.2337/ db09-1011

62. Kong J, Chen Y, Zhu G, Zhao Q, Li JC. 2013. 1,25-Dyhydroxyvitamin D3 upregulates leptin expression in mouse adipose tissue. J Endocrinol
216(2): 265-271. doi: https://doi.org/10.1530/JOE-12-0344

63. Indulekha K, Anjana RM, Surendar J, Mohan V. 2011. Association of visceral and subcutaneous fat with glucose intolerance, insulin resistance, adipocytokines and inflammatory markers in Asian Indians (CURES-113). Clin Biochem 44(4): 281-287. doi: https://doi. org/10.1016/j.clinbiochem.2010.12.015

64. Karonova T, Belyaeva O, Jude EB, Tsiberkin A, Andreeva A, et al. 2016. Serum 25(OH)D and adipokines levels in people with abdominal obesity. J Steroid Biochem Mol Biol (In press). doi: https://doi. org/10.1016/j.jsbmb.2016.09.005

65. Maetani M, Maskarinec G, Franke AA, Cooney RV. 2009. Association of leptin, 25-hydroxyvitamin D, and parathyroid hormone in women. Nutr Cancer 61(2): 225-231. doi: https://doi. org/10.1080/01635580802455149

66. Ohashi K, Ouchi N, Matsuzawa Y. 2012. Anti-inflammatory and antiatherogenic properties of adiponectin. Biochimie 94(10): 2137-2142. doi: https://doi.org/10.1016/j.biochi.2012.06.008.

67. Nimitphong H, Chanprasertyothin S, Jongjaroenprasert W, Ongphiphadhanakul B. 2009. The association between vitamin D status and circulating adiponectin independent of adiposity in subjects with abnormal glucose tolerance. Endocrine 36(2): 205-210. doi: https://doi. org/10.1007/s12020-009-9216-9

68. Roth CL, Kratz M, Ralston MM, Reinehr T. 2011. Changes in adiposederived inflammatory cytokines and chemokines after successful lifestyle intervention in obese children. Metabolism 60(4): 445-452. doi: https://doi.org/10.1016/j.metabol.2010.03.023

69. Anderson JL, May HT, Horne BD, Bair TL, Hall NL, et al. 2010. Relation of vitamin D deficiency to cardiovascular risk factors, disease status, and incident events in a general healthcare population. Am J Cardiol 106(7): 963-968. doi: https://doi.org/10.1016/j. amjcard.2010.05.027 